\title{
1 Stochastic Petri net-based modelling of the durability of renderings
}

\section{Ferreira ${ }^{1}$, L. Canhoto Neves ${ }^{2}$, A. Silva ${ }^{3}$ and J. de Brito ${ }^{4}$}

3

\section{Abstract:}

6 In this study, a methodology to model and predict the life-cycle performance of building

7 façades based on Stochastic Petri Nets is proposed. The proposed model evaluates the

8 performance of rendered façades over time, evaluating the uncertainty of the future

9 performance of these coatings. The performance of rendered façades is evaluated based on a discrete qualitative scale composed of five condition levels, established according to

11 the physical and visual degradation of these elements. In this study, the deterioration is

12 modelled considering that the transition times between these condition states can be

13 modelled as a random variable with different distributions. For that purpose, a Stochastic

14 Petri Nets model is used, as a formal framework to describe this problem. The model's validation is based on probabilistic indicators of performance, computed using MonteCarlo simulation and the probability distribution parameters leading to better fit are defined as those maximizing the likelihood, computed using Genetic Algorithm. In this study, a sample of 99 rendered façades, located in Portugal, is analysed, and the degradation condition of each case study is evaluated through in-situ visual inspections. The model proposed allows evaluating: i) the transition rate between degradation conditions; ii) the probability of belonging to a given degradation condition over time; and iii) the mean time of permanence in each degradation condition. The use of Petri Nets shows to be more accurate than a more traditional approach based on Markov Chains, but

\footnotetext{
${ }^{1}$ PhD Student, FCT - UNL (Faculty of Sciences and Technology - New University of Lisbon), 2829-516, Caparica, Portugal, c.ferreira@ campus.fct.unl.pt

2 Assistant Professor, Nottingham Transport Engineering Centre (NTEC), University of Nottingham, Nottingham, UK, Luis.Neves@ nottingham.ac.uk

${ }^{3}$ Postdoctoral researcher, IST - University of Lisbon, Av. Rovisco Pais, 1049-001, Lisbon, Portugal, email: anasilva931@msn.com, Corresponding author

${ }^{4}$ Full Professor, Department of Civil Engineering and Architecture, IST - University of Lisbon, Av. Rovisco Pais, 1049-001, Lisbon, Portugal, e-mail: jb@civil.ist.utl.pt
} 
24 also allows developing future research to consider different environmental conditions,

25 maintenance actions or inspections, amongst other aspects of life-cycle analysis of 26 existing assets.

27

28 Keywords: Petri nets; rendered façades; genetic algorithms; degradation. 


\section{Introduction}

30 According to Jensen and Rozenberg (2012), the net theory can be seen as "a system theory that aims at understanding systems whose structure and behaviour are determined by a combinatorial nature of their states and changes". The first proposal of nets of places and transitions, proposed by C. A. Petri (Petri, 1962), allows developing a non-idealizing methodology to concurrency and information flow, in organizational systems (Genrich and Lautenbach, 1981). Petri nets are considered a mathematical and graphical tool for the formal description of systems whose dynamics are characterized as being concurrent, asynchronous, distributed, parallel, nondeterministic, and/or stochastic, mutual exclusive, and conflicting, which are typical features of distributed environments (Murata, 1989). Therefore, Petri nets allow capturing the static and the dynamic nature of a real system, thus characterizing the rate of transition between states or conditions (Marsan et al., 1994).

Due to their characteristics, Petri nets have been successfully applied in different fields of knowledge, namely in robotics (Al-Ahmari, 2016), in the optimization of manufacturing systems (Chen et al., 2014; Uzam et al., 2015), business process management (van der Aalst, 2002), human computer interaction (Tang et al., 2008), among others. Petri nets are not widely used in the construction industry, and particularly in building asset modelling. Nevertheless, there are various works (Li, 1998; Cheng et al., 2011; Molinero and Núñez, 2011; Cheng et al., 2013; Rinke et al., 2017) that use Petri nets to manage resources, to estimate equipment availability and scheduling of tasks on the site-work during the building design process. On the other hand, recent work has been published on the use of Petri Nets to model the deterioration of other civil engineering infrastructures (Andrews, 2013; Rama and Andrews, 2013; Le and Andrews, 2015; Le and Andrews, 2016; Leigh and Dunnett, 2016; Yianni et al., 
54 2017; Zhang et al., 2017). In the last decades, various authors proposed several 55 extensions and adaptations of ordinary Petri nets; all of them based on the basic Petri net formalism, but presenting very different characteristics and assumptions, in order to adapt themselves to the phenomena under analysis. Consequently, there is a reasonable expertise in the application of Petri nets to different application domains, thus allowing transferring knowledge and methodologies from one field to another (Girault and Valk, 60 2002).

61 This study intends to evaluate the suitability and advantages of the use of Stochastic 62 Petri Nets (SPN) as deterioration models in building asset management. The main advantages of SPN are their graphical representation, allowing a better and more intuitive understanding of the modelling principles, and their versatility, allowing the modelling complex stochastic processes. In the particular case of deterioration modelling, and compared to the more traditional Markov Chains, SPN allow the seamless use of different probabilistic distributions. Furthermore, their versatility allow modelling, in a common framework, multiple aspects of asset management, including deterioration, maintenance, inspection, and decision-making. In this study, a model to predict the life-cycle performance of building façades based on stochastic Petri nets is proposed. To analyse the degradation condition of rendered façades over time, a set of Petri net models considering different probabilistic distributions are used to estimate the transitions times between condition levels. Since there are no closed form expressions for the probability distribution of the condition state at a certain time, Monte Carlo simulation is used to compute the likelihood of each model. However, the errors introduced by Monte Carlo simulation require the use of gradient-independent optimization methods, like Genetic Algorithms, to identify the optimal parameters of the probability distributions. 
The sample analysed in this study comprises 99 renderings, located in Portugal, for which degradation condition was evaluated through in situ visual inspections. The classification system adopted in this study to evaluate the deterioration state of rendered façades is a discrete qualitative scale divided in five condition levels, proposed by Gaspar and de Brito (2008, 2011), ranging between "no visible degradation" (condition A) and "generalised degradation" (condition E), which requires an immediate rehabilitation or maintenance action.

In the first part of this study, a traditional method, based on Markov chains is applied, in order to define a benchmark model. The benchmark model and the Petri net model with transition times exponentially distributed are used to validate the methodology proposed. The comparison of the models is possible since the stochastic Petri net with transitions exponentially distributed is equivalent to a finite Markov chain. After that, a

91 set of probabilistic distributions are used to analyse the degradation condition of

92 rendered façades over time. The information obtained from the Petri net models allows

93 the identification of the degradation rate of rendered façades, characterizing the pattern

94 that characterizes the loss of performance of these claddings over time. This information is crucial to identify the future need for interventions, optimizing the maintenance

96 needs, and thus avoiding unnecessary cost associated with urgent interventions.

97 The outline of this paper is as follows: Section 2 provides a literature review concerning 98 the classification system and modelling techniques used to model the evolution of the 99 degradation in rendered façades; Section 3 introduces the concept of Petri nets, as well 100 as the procedure used to predict the life-cycle performance of renderings. Finally, the 101 discussion of the results is presented in Section 4 and conclusions are drawn in Section 102 5. 


\section{Literature review}

104 The façades can be seen as the skin of the building, i.e. they can be considered the first 105 layer of protection against the deterioration agents (Silva et al., 2015), thus being the 106 element more prone to degradation. According to Flores-Colen and de Brito (2010) the 107 claddings' degradation level can influence the quality of the urban environment, since it

108 affects the architectural appearance of buildings, which has a considerable effect on the 109 physical comfort of inhabitants of larger cities (Korjenic et al., 2016). Rendered façades

110 are the most common type of cladding in Portugal (Census, 2001). In the present

111 context of societies aiming at achieving a more sustainable use of resources, it is 112 increasingly important to define rational maintenance strategies so as to avoid 113 unnecessary costs (Wang and Xie, 2002; Arain and Pheng, 2006; Wong and Li, 2009).

114 For that purpose, it is essential to develop new and versatile tools to support the 115 decision-making process regarding the instant in which maintenance actions must be 116 performed, knowing the degree of uncertainty associated with the estimates (Frangopol,

117 2011). To achieve this, the present work focuses on the use of probabilistic based 118 methods for modelling performance, including Stochastic Petri Nets and Markov 119 Chains.

120 The definition of maintenance strategies is, in general, related with the users' demands,

121 i.e. more demanding users may demand a high level of performance, requiring that the 122 cladding be replaced as soon as it starts to deteriorate; on the other hand, some users 123 may accept a lower level of performance, thus minimizing the maintenance costs 124 (Shohet et al., 1999). Consequently, the definition of maintenance strategies requires the 125 condition assessment of rendered façades and the knowledge of their expected service 126 life. According to Hertlein (1999), condition-based maintenance by inspection planning 127 can be a useful tool to reduce the life cycle costs, achieving a more rational and efficient 
way to manage maintenance budgets (Flores et al., 2011).

129 In the last decades, different studies (Shohet et al., 2002; Shohet and Paciuk, 2004;

130 Gaspar and de Brito, 2008; Paulo et al., 2014; Paulo et al., 2016) propose visual and

131 physical scales to characterize the type, extension and severity of defects observed in

132 rendered façades. Gaspar and de Brito (2008) and Silva et al. (2014) proposed a discrete

133 scale to evaluate the degradation condition of rendered façades (Table 1).

134 This qualitative scale, based on the evaluation of the physical and visual degradation of 135 rendered façades analysed during a comprehensive fieldwork, can be associated with a

136 quantitative index that portrays the global performance of the façades. This numerical

137 index, initially proposed by Gaspar and de Brito $(2008,2011)$, expresses the global

138 degradation of façade coatings through the ratio between the degraded area weighted as

139 a function of its condition and a reference area, equivalent to the whole and having the

140 maximum degradation level possible - equation (1).

141

$$
S_{w}=\frac{\sum\left(A_{n} \times k_{n} \times k_{a, n}\right)}{A \times k}
$$

142 Where $S_{w}$ is the degradation severity of the coating, expressed as a percentage; $k_{n}$ is the

143 multiplying factor of anomaly $n$, as a function of their degradation level, within the

144 range $K=\{0,1,2,3,4\} ; k_{a, n}$ is a weighting factor corresponding to the relative weight

145 of the anomaly detected $\left(k_{a, n} \in \mathrm{R}+\right) ; k_{a, n}=1$ by default; $A_{n}$ is the area of coating affected

146 by an anomaly $n ; A$ is the façade area; and $k$ is the multiplying factor corresponding to

147 the highest degradation level of a coating of area $A$.

148 In this study, the anomalies that occur in rendered façades are grouped in three

149 categories: stains; cracking; and detachment. The coefficient $k_{a, n}$ allows establishing a

150 relative weight between these groups of anomalies, based on the cost of repair of each 
151 anomaly, its aesthetic impact, the influence on the renderings' service life, the

152 fulfilment of performance requirements (e.g. watertightness) and its propensity to

153 generate new anomalies. In this study, the following $k_{a, n}$ values are adopted for the

154 different groups of anomalies: 1.0 for cracking; 1.5 for detachment; and 0.25 for stains

155 in condition B and 0.67 for stains in more serious condition levels (C, D and E).

156 Figure 1 shows the correlation between the condition of some façades inspected and the

157 numerical index, illustrating the visual conditions of rendering in each degradation

158 condition.

159 2.1 Application of Markov chains to model the evolution of the degradation of

160 rendered façades

161 Markov chains are widely used by researchers in several fields of civil engineering (Wang

162 and Zaniewski, 1996; Hawk and Small, 1998; Thompson et al., 1998; McDulling, 2006;

163 Ortiz-García et al., 2006). Particularly, continuous-time Markov chains are commonly

164 used in modelling the deterioration of civil engineering assets (Kallen and van Noortwijk,

165 2006). This modelling technique is considered an intuitive, simple and computationally

166 inexpensive stochastic process, since analytical solutions exist and the memoryless

167 property allows estimating the future performance only based on the current performance,

168 becoming particularly relevant when limited information is available.

169 Silva et al. (2015) used continuous-time Markov chains to evaluate the degradation

170 process of external render over time, based on the visual inspections of characteristics and

171 condition of façades located in Portugal. In this work, it is assumed that the progression of

172 damage is continuous and, over a small time interval, the condition of the façade can only

173 remain constant or deteriorate to the next condition state. The intensity matrix defines the

174 rate of transitions between states (Kalbfleisch and Lawless, 1985) as: 
$175 \quad \mathbf{Q}=\left[\begin{array}{ccccc}-q_{1,2} & q_{1,2} & \cdots & 0 & 0 \\ 0 & \ddots & \ddots & 0 & 0 \\ \vdots & \ddots & \ddots & \ddots & \vdots \\ 0 & 0 & \ddots & -q_{n-1, n} & q_{n-1, n} \\ 0 & 0 & \cdots & 0 & 0\end{array}\right]$

176 The estimation of the optimal intensity matrix, leading to the best fit between the model

177 and the observed condition, was based on the concept of maximum likelihood described

178 by Kalbfleisch and Lawless (1985). Likelihood is defined as the predicted probability of

179 occurrence of the observed transitions:

$180 \quad L(\mathbf{Q})=\prod_{i=1}^{m} \prod_{j=1}^{k} p_{i j}$

181 Where $i$ is the condition level in the initial instant, $j$ is the condition level in the final 182 instant, $m$ is the number of elements, $k$ is the number of intervals between inspections, 183 and $p_{i j}$ is the probability of transition from condition level $i$ to condition level $j,(i, j)$ 184 entry of the transition probability matrix, $\mathbf{P}$, given by:

$185 \mathbf{P}(\Delta t)=e^{\mathbf{Q} \cdot \Delta t}$

186 Where $\Delta t$ is the time interval between inspections.

187 The optimization of the intensity matrix, $\mathbf{Q}$, was performed using the active set algorithm 188 implemented in MATLAB $^{\circledR}$. The aim of the optimization algorithm is to find the 189 parameters of the intensity matrix, $\mathbf{Q}$, which maximize the fitness function, $\log V=$ $190 \max \left(\sum \sum \log p_{i j}\right)$, while keeping all terms of matrix $\mathbf{Q}$ positive, $q_{i-1, i}>0$ where

$191 i \in\{2, \ldots, n\}$. This optimization algorithm is a reasonable tool for problems that use 192 analytical expressions. In situations where analytical expressions are not available, the 193 numerical estimation of the functions can lead to convergence problems and lack of 194 robustness of the solution.

\section{Petri nets}




\subsection{Conventional Petri nets}

197 The concept of Petri nets was originally present by Carl A. Petri, who in his doctoral 198 thesis developed a new model of information flow and control in systems (Petri, 1962).

199 Petri nets are a graphical and mathematical modelling tool, suitable for characterizing 200 concurrent, asynchronous, distributed, parallel, nondeterministic, and/or stochastic 201 systems (Murata, 1989).

202 An ordinary Petri net is considered a directed, weighted, bipartite graph with an initial 203 state called the initial marking, $M_{0}$ (Murata, 1989; Schneeweiss, 2004). It is called a 204 bipartite graph because nodes are divided into two different types: places, usually 205 represented by circles, and transitions, usually represented by rectangles. Both nodes are linked by directed arcs, from places to transitions (input arcs) and from transitions to 207 places (output arcs) (Peterson, 1977; Murata, 1989; Schneeweiss, 2004). The third 208 element of a Petri nets are tokens, usually represented by black dots, which represent the 209 elements in the system (Peterson, 1977; Murata, 1989). Figure 2 shows a simple Petri net model. Transition $T_{1}$ has two input places $\left(P_{1}\right.$ and $\left.P_{2}\right)$ and one output place $\left(P_{3}\right)$. The

211 arcs that connect the input places to the transition and the transition to the output place 212 represent the pre- and post-conditions of the transition, respectively. When all input 213 places are occupied by a token the transition is said to be enabled. At this point, the 214 transition fires, the tokens are removed from the input places, and new tokens are created 215 in the output places. In this example, transition $T_{1}$ is not enabled because the pre216 conditions of the transition are not complied with, i.e. there is a token in place $P_{1}$, but no 217 token at place $P_{2}$. Once tokens exist in both $P_{1}$ and $P_{2}$, transition $T_{1}$ will fire, tokens 218 from places $P_{1}$ and $P_{2}$ will be removed and a token will be placed in place $\mathrm{P}_{3}$.

219 In the context of this study, places represent resources or conditions while transitions can 220 represent actions or events that cause the system to change (Murata, 1989). Tokens are 
221 stored in places representing the present state of the system and transitions allow the

222 tokens to move between two places modelling, in this way, the dynamic behaviour of the 223 system.

224 When analysing a PN, conflicts might occur when two or more transitions are enabled 225 from a common place and the firing of one transition disables the other transitions 226 (Bowden, 2000). In the literature, there are several proposals for solving conflicts, either 227 deterministically, for example through the introduction of a priority transition by the 228 user, or probabilistically, by assigning probabilistic properties to the conflicting 229 transitions (David and Alla, 2010; Wang, 2012). However, in timed Petri nets, the most 230 common way to solve conflicts is through firing times, assuming that the transition with 231 the shortest delay will fire first (Murata, 1989).

\section{$232 \quad 3.2$ Stochastic Petri net}

233 In the original definition of Petri nets, the concept of time is not explicitly included (Murata, 234 1989). However, many applications are time dependent and the introduction of time delays 235 has to be considered. The notion of time in Petri nets was initially introduced by 236 Ramamoorthy and Ho (1980) and Zuberek (1980). In these two works, deterministic time 237 intervals are used for each transition, creating a delay between the instant the transition 238 becomes enabled and the firing instant. Molly (1982) introduced the concept of stochastic 239 Petri net by assigning an exponentially distributed firing rate to each transition for 240 continuous time systems. After that, several classes of stochastic Petri nets have been 241 proposed for performance and reliability analysis of systems, the more relevant of which

242 are: the generalized stochastic Petri net (Marsan et al., 1984), the extended stochastic Petri 243 net (Dugan et al., 1984), and the deterministic and stochastic Petri net (Marsan and Chiola, 244 1987). 
245 The model employed in this work considers Petri nets with transitions times defined as

246 a random variable, as proposed by Molly (1982). However, the results obtained showed

247 that the exponential distribution for the firing times, proposed by Molly (1982), were

248 not always adequate. To overcome this limitation, the proposal of Dugan et al. (1984),

249 allowing any probability distribution to be used to model the firing rate was used.

250 Mathematically, the theory behind the stochastic Petri net is the same as the Petri net;

251 their mode of operation is identical, applying the same firing rules. The only difference

252 is the random time interval between the transition becoming enabled and firing.

\section{$253 \quad 3.3$ Deterioration Petri net model}

254 Deterioration can be modelled with Petri nets by considering that each place is a condition state of the classification system adapted, tokens indicate the current condition of an element, and timed transitions define the movement between condition states (Le, 2014, Yianni et al., 2016, 2017). In this work, a five condition levels Petri net scheme is defined. Since maintenance actions are not considered, the condition level of the infrastructure deteriorates continuously over time until it reaches the worst condition level defined in the performance scale.

261 The time dependent nature of the problem is included by defining timed transitions. The time specified by each transition represents the sojourn time in the condition level, i.e., the

263 time that infrastructure spends in condition level $i$ before moving to condition level $i+1$.

264 The timed transitions are modeled by probability distributions.

\section{$265 \quad 3.4$ Parameter estimation}

266 The probability distribution that best describes the deterioration process of an

267 infrastructure is that resulting in higher probabilities of occurrence of observed transitions. 
268 In order to identify the parameters of the probability distribution that provide a best fit,

269 parameter estimation is required. The parameters of the probability distribution are fitted

270 to historical data through the maximum likelihood method proposed by Kalbfleisch and

271 Lawless (1985) and shown in equation (3). To simplify the computations and improve

272 robustness, the logarithm of the likelihood is maximized.

\section{$273 \quad$ 3.4.1 Monte Carlo simulation}

274 The probability of occurrence of the observed transition, $p_{i j}$, is estimated by Monte Carlo

275 simulation. This is a helpful approach to compute numerical approximations in situations

276 where it is not feasible to obtain analytical solutions and can be used to consider the

277 propagation of uncertainties during the lifetime of the infrastructure. This method allows

278 generating random sojourn times to each condition level from the inverse CDF

279 (cumulative distribution function) of probability distribution.

280 The proposed procedure for computing the probability of occurrence of the observed

281 transition, $p_{i j}$, is illustrated in Figure 4. The procedure depicted is repeated for each

282 transition observed in the historical database. The input for the algorithm includes the

283 information about each observed transition: time interval between observations, $\Delta t$,

284 condition level in the initial instant, $i$, and condition level in the final instant, $j$. The

285 condition level in the initial instant, $i$, is used to define the initial marking, $M_{0}$, of the Petri

286 net, the time interval between observations, $\Delta t$, is the time horizon of the analysis, and the

287 condition level in the final instant, $j$, is used to compute the probability of occurrence at

288 the end of the procedure. The first transition to fire is identified by checking which

289 transitions are enabled. When more than one transition is enabled, the transition with less

290 time delay is the first to fire. However, since the Petri net defined for the deterioration

291 model is arranged in sequential manner and there is only one token in the Petri net, i.e. 
292 conflicts do not need to be considered. In the next analysis step, the sojourn time in the

293 initial condition level is computed, and the Petri net and time are updated. The process is

294 repeated until $\Delta t$ is reached. The output of the procedure is the condition index at the time

295 horizon for each sample. Using Monte-Carlo simulation the distribution of the final

296 condition can be computed and the probability of the observed transition occurring can be

297 calculated.

\section{$298 \quad$ 3.4.2 Optimization}

299 The optimization of the parameters of the probability distributions is performed using

300 Genetic Algorithms (GA), which were selected for being widely available, robust and

301 efficient for objective functions computed using Monte-Carlo simulation. In fact, by

302 using only information on the objective function, not requiring the computation of

303 gradients, GA avoid the potential consequences of numerical errors, significantly

304 simplifying the problem (Man et al, 1999; Morcous and Lounis, 2005).

305 The GA used for optimization of the parameters of the probability distributions is 306 simply depicted in Figure 5. The optimization procedure begins with the definition of 307 optimization variables, objective function, and constraints. The objective function is 308 used to measure the degree of "goodness" of each individual of the population (Man et 309 al, 1999; Morcous and Lounis, 2005). All parameters of the probability distributions are

310 defined as problem parameters, and the Monte-Carlo procedure described above is used 311 to compute the objective function.

312 In the following step, the initial population is randomly generated. A population is 313 composed by a set of individuals, where each individual is a potential solution of the

314 problem. All individuals of the initial population are evaluated through the objective 315 function, where the best individual is the one with the highest value of the likelihood. At 
316 each step of the optimization process, the GA uses the best individual of the current

317 population to create the offspring generation (MatLab, 2016), using the crossover and

318 mutation procedures. The new population generated is then evaluated using the objective

319 function and used as a new parent population. This process is repeated iteratively until a

320 predefined stopping criteria is satisfied.

321 In this study, the optimization of the parameters of the probability distributions was

322 performed using the GA available in MATLAB $^{\circledR}$ (MatLab, 2016). The parameters used in

323 the GA are the following:

- Size of the population: 50 individuals when the number of optimization variables is less than or equal to 5; and 200 individuals otherwise;

- Stopping criteria: the algorithm stops if the average relative change in the best fitness function value over 50 generations (minimum number of generations) is less than or equal to $10^{-6}$;

- Mutation procedure was performed using the Gaussian algorithm implemented

In the extension of Petri nets proposed by Molly (1982), the stochastic sojourn time is modelled as an exponentially distributed random variable. In this case, a stochastic Petri net is isomorphic to a finite Markov chain.

\section{APPLICATION TO RENDERED FAÇADES}

335 The deterioration Petri net model for façades is illustrated in Figure 3. It is composed of five

336 places $C_{1}$ to $C_{5}$ each representing one of the five discrete states that characterize the

337 degradation condition of external render façades defined in section 2. Level A means there 338 is no visible degradation and Level $\mathrm{E}$ indicates the presence of extensive damage in the 339 render façade. The transitions $T_{1}$ to $T_{4}$ represent the time interval required for the façades to 
progress to a more deteriorated state.

341 Since Markov chains are widely used to evaluate the condition level over time and taking

342 into account the isomorphism between Markov chains and stochastic Petri nets, the Petri

343 net model proposed can be validated by comparison with the Markov chains model

344 proposed by Silva et al. (2015). In this manner, the efficiency of the numerical procedure

345 and the optimization algorithm described in section 3.4 can be evaluated.

346 The data presented by Silva et al. (2015) is therefore used to calibrate both the Markov

347 chain model and the Petri net models. The database is composed of 99 visual inspection

348 records of external render façades located in Portugal. For each façade, only the initial

349 condition level (assuming that at time zero the render is in Level A) and final condition,

350 corresponding to the inspection date, are known.

\section{$351 \quad 4.1$ Validation of the Petri net model}

352 In Table 2 the optimal transition rates considering a Markov chain model, implemented

353 using analytical expressions, and a Petri Net model with exponentially distributed sojourn

354 times, are compared. The values of the parameters for each condition level are quite

355 similar as expected. The differences obtained are due to sampling errors associated with

356 the Monte-Carlo simulation used in conjunction with the Petri Net model (Figure 6).

357 Table 3 shows the number of observed and predicted façade in each condition level for

358 both models. The results show that both models are suitable to model the deterioration 359 process of the external façade renders. The biggest relative error is obtained for the Level

360 D (15.3\% for Markov chains and $16.5 \%$ for Petri nets).

361 Taking into account the results obtained by Petri nets, it is confirmed that the proposed model is suitable to evaluate the degradation of external façade renders. 


\subsection{Probabilistic analysis}

364

365

366

367

368

369

370

371

372

374

375

376

377

378

379

380

381

382

\subsubsection{Two-parameter distributions}

When using Petri net models, in addition to the exponential distribution, four distributions were studied: Weibull, Lognormal, Gumbel, and Normal. Table 4 shows the optimal parameters obtained in all probability distribution analysed as the likelihood computed for each set of optimal parameters. All the studied distributions lead to a better likelihood than the exponential distribution.

Table 5 shows the number of observed and predicted façades in each condition level for each probability distribution and Table 6 shows the relative error obtained for each case. The values obtained for the relative error are low and, in all cases, acceptable; the largest errors occur for the exponential distribution. Amongst the alternative distributions, the largest error is associated with the Gumbel distribution in Level A (8.6\%). The results in those two tables show that the exponential distribution is the one with the largest mean relative error for all states $(7.0 \%)$, while the smallest mean relative error for all states is for the lognormal distribution $(2.1 \%)$. The normal distribution presents a mean relative error for all states of $3.3 \%$ (second lower value).

Figure 7 presents the average predicted condition profile of the external render façade over time for each probability distribution analysed. The profiles obtained for the four distributions are showing some differences to the profile obtained for the exponential distribution.

The deterioration curve obtained by exponential distribution does not have inflection points (concave up). The other distribution curves have two inflection points (Figure 7a). In the transition between levels $\mathrm{B}$ and $\mathrm{C}$ there is an inflection point, where the concavity of the curve changes. The second inflection point occurs between levels $\mathrm{C}$ 
387 and D. In terms of dispersion of the results (Figure $7 \mathrm{~b}$ ), any of the distributions

388 (Weibull, Lognormal, Gumbel, Normal) has lower dispersion values over the simulated 389 period than the exponential distribution. In fact, the exponential distribution has a mean 390 value equal to the standard deviation. There is no physical reason indicating this occurs 391 for the sojourn times. As a result, the use of Markov chains has limited ability to model 392 the variability of performance, frequently overestimating it.

393 These differences between the degradation curves also have high impact on the 394 probabilistic distribution of the degradation condition level over time (Figure 8a-c). 395 Despite the peaks occurring, approximately, in the same years, their values are quite 396 different.

397 For level A, the predicted probabilities for all distributions are similar, beginning with 398 probability equal to 1 and decreasing rapidly over time; at year 10 the probability of a 399 render façade being in level A is near zero (Figure 8a). Also, for level B (Figure 8a), the 400 predicted probabilities for all distributions are similar; the maximum probability of a 401 façade belonging to level $\mathrm{B}$ occurs between years 3 and 4; after that, the value of the 402 probability decreases rapidly. In level C significant differences can be observed between 403 models (Figure $8 \mathrm{~b}$ ). The maximum probability of belonging to level $\mathrm{C}$ is close to 0.50 for 404 the exponential distribution while for the other distributions it varies between 0.70 and 405 0.80. After the maximum probability is achieved, the slope of the exponential distribution 406 is softer, when compared with the other distributions. For level D (Figure 8b), the 407 exponential distribution has a smoother growth when compared to other distributions, 408 then the peak occurs in all distributions between years 18 and 19 (the maximum 409 probability of belonging to level $\mathrm{D}$ is close to 0.40 for the exponential distribution while 410 for the other distributions it varies between 0.70 and 0.80 ). After that, the slope of the 411 exponential distribution is softer. Finally, as expected, the probability of belonging to 
412 level E (Figure 8c) increases over time; however, the increase for the exponential

413 distribution is softer than for the other distributions. At year 40, for the other distributions,

414 the probability of a façade belonging to level $\mathrm{E}$ is bigger than 0.95 while, for the

415 exponential distribution, it is closer of 0.80 .

416 In the analysis of the service life and durability of rendered façades, it is assumed that level

417 D corresponds to the end of the service life of rendered façades, beyond which a 418 maintenance action must be performed. Figure $8 \mathrm{~b}$ shows the probabilistic distribution of the 419 degradation condition $\mathrm{D}$ over time. The results reveal that the exponential models and, 420 consequently, the Markov chain models, are less accurate in predicting the behaviour of 421 deteriorated serious conditions, due to the reduced number of samples available. According 422 to the Markov chain model proposed by Silva et al. (2015), the probability of a rendering belonging to level $\mathrm{D}$ reaches a peak at 15 years. In this study, using a Petri net model, this

424 peak is between 18 and 19 years. These values seem coherent with physical reality, in 425 agreement with the results present in the literature: i) Shohet et al. (1999) obtained an expected service life for cementitious renders of 20 years; ii) Shohet and Paciuk (2004) estimated a predicted service life of 15 years for a stricter level of demand (with a range of results between 12 and 19 years), and a service life of 23 years (with a range of results between 19 and 27 years) for a lower level of demand; iii) Mayer and Bourke (2005) 430 obtained an estimated service life of 18 years for current renderings; iv) Gaspar and de Brito 431 (2008) estimated a service life of cement-rendered façades of 22 years; v) Silva et al. 432 (2013), using an artificial neural network model, obtained an estimated service life of 22 433 years with a 16-28 years range, and using a multiple linear regression model, obtained an 434 average estimated service life of 15 years with a range between 12 and 17 years; vi) a 435 comparative analysis of service life prediction methods applied to rendered façades (Silva et 436 al. 2016), led to an average value of the estimated service life of rendered façades ranging 


\subsubsection{Three-parameter distributions}

439 The results of the previous section show that the probability distributions with two

440 parameters show a better fit to the historical data when compared with the exponential 441 distribution. In an attempt to examine whether a probability distribution with three 442 parameters is an option to better model the degradation of façades over time, the Weibull 3-

443 parameters distribution was used. The probability density function of this distribution is 444 given by:

$445 \quad f(t \mid \alpha, \beta, \gamma)=\frac{\beta}{\alpha}\left(\frac{t-\gamma}{\alpha}\right)^{\beta-1} e^{-\left(\frac{t-\gamma}{\alpha}\right)^{\beta}}$

446 where $\alpha, \beta$ has the same definition given in Table 4 and $\gamma \in \mathbb{R}$ is the location parameter 447 of the distribution.

448 The optimal parameters obtained for Weibull 3-parameters distribution and the likelihood 449 obtained for this set of parameters are shown in Table 7. Table 8 shows the number of 450 observed and predicted façades in each condition level.

451 From the analysis of the results obtained in the two-parameters distribution (Tables 4 452 and 5) and the three-parameters distribution (Tables 7 and 8), it is found that the 453 Weibull 3-parameters shows a better fit than the two-parameters distribution, both in 454 terms of likelihood and mean relative error. However, the Weibull 3-parameters 455 distribution increases the level of complexity of the analysis (the number of parameters 456 to be optimized increase from 8 to 12 ). 


\section{CONCLUSIONS}

458 In this paper, a model to asses and predict the life-cycle performance of building

459 façades based on stochastic Petri nets is proposed. The application of Petri nets to

460 degradation models is a recent research field, but this modelling technique has shown

461 several advantages relative to the more traditional Markov chains. The graphical

462 representation can be used to describe the problem in an intuitive way; PN are more

463 flexible than the Markov chains, allowing the incorporation of a multitude of rules in

464 the model to accurately simulate complex situations and keeping the model size within

465 manageable limits. Moreover, with this modelling technique, transition times are not 466 required to be exponential distributed.

467 The sojourn time is defined as a random variable for each condition level. The

468 deterioration rates were estimated from available historical data, based on the analysis of 469 the degradation condition of 99 rendered façades, located in Portugal. The Petri net model

470 with transition times exponentially distributed was used to validate the methodology

471 proposed by comparison with a benchmark model based on Markov chains. In order to

472 investigate whether other probability distributions would fit the historical data better than

473 the exponential distribution, five probability distributions were analysed using Petri net

474 models (Weibull 2-parameters, Weibull 3-parameters, Lognormal, Gumbel, and Normal).

475 From the results of the probabilistic analysis performed with Petri nets model, it was

476 found that the use of distributions with two parameters greatly improves the model's

477 goodness of fit. The likelihood values of the four distributions (Weibull 2-parameters,

478 Lognormal, Gumbel, and Normal) are quite similar and all significantly better than the

479 exponential distribution. Some improvement is obtained when a Weibull 3-parameters

480 distribution in considered, but this is obtained at the expense of a significant increase in 
the complexity of the model.

482 In this study, the degradation condition of rendered façades is described by five condition

483 states, ranging between A (most favourable, without visible degradation) and $\mathrm{E}$ (most

484 serious, with generalised degradation). For the analysis of the service life and durability of 485 rendered façades, it is assumed that level D corresponds to the end of the service life of 486 rendered façades, beyond which a maintenance action must be performed. Based on the 487 Petri net model proposed, a rendered façade presents the higher probability of reaching the 488 end of its service (corresponding to level D) between 18 and 19 years. The results obtained 489 are consistent with physical reality and in agreement with the results present in the 490 literature. This study evaluates the loss of performance of rendered façades over time, 491 modelling the probability of transition between degradation conditions through Petri net

492 models. This study demonstrates the validity of this approach to model the degradation of 493 external claddings and, therefore, in future studies, the authors intend to apply a similar 494 methodology to predict the service life of other cladding systems, encompassing the effects 495 of their characteristics in their degradation process (e.g. environmental exposure 496 conditions).

\section{ACKNOWLEDGEMENTS}

498 The authors gratefully acknowledge the support of the CERIS-ICIST Research Institute, 499 IST, University of Lisbon and the FCT (Foundation for Science and Technology) through 500 program SFRH/BD/88195/2012.

\section{REFERENCES}

502 Al-Ahmari, A. (2016) Optimal robotic cell scheduling with controllers using 503 mathematically based timed Petri nets. Information Sciences, 329, 638-648. 
504 Andrews, J. (2013). A modelling approach to railway track asset management.

505 Proceedings of the Institution of Mechanical Engineers, Part F: Journal of Rail and 506 Rapid Transit, 227(1), 56-73.

507 Arain, F. M., \& Pheng, L. S. (2006). Knowledge-based decision support system for 508 management of variation orders for institutional building projects. Automation in 509 Construction, 15(3), 272-291.

510 Bowden, F. D. J. (2000). A brief survey and synthesis of the roles of time in Petri nets.

511 Mathematical and Computer Modelling, 31(10):55-68.

512 Chen, Y., Li, Z., Barkaoui, K. (2014) Maximally permissive liveness-enforcing 513 supervisor with lowest implementation cost for flexible manufacturing systems. 514 Information Sciences, 256, 74-90.

515 Cheng, F., Li, H., Wang, Y. W., Skitmore, M., \& Forsythe, P. (2013). Modeling 516 resource management in the building design process by information constraint Petri 517 nets. Automation in Construction, 29, 92-99.

518 Cheng, F. F., Wang, Y. W., Ling, X. Z., \& Bai, Y. (2011). A Petri net simulation model 519 for virtual construction of earthmoving operations. Automation in Construction, 20(2), $520 \quad 181-188$.

521 David, R. and Alla, H. (2010). Discrete, continuous, and hybrid Petri nets. Springer 522 Science \& Business Media.

523 Dugan, J.B., Trivedi, K.S., Geist, R.M. \& Nicola, V.F. (1984) Extended stochastic Petri 524 nets: applications and analysis. Technical report, Duke University, Durham, NC, USA.

525 Flores-Colen, I., \& de Brito, J. (2010). A systematic approach for maintenance 
526 budgeting of buildings façades based on predictive and preventive strategies.

527 Construction and Building Materials, 24(9), 1718-1729.

528 Flores-Colen, I., de Brito, J., Freitas, V.P. (2011) On-site performance assessment of 529 rendering façades for predictive maintenance. Structural Survey, 29(2), 133-146.

530 Frangopol, D. M. (2011). Life-cycle performance, management, and optimization of 531 structural systems under uncertainty: accomplishments and challenges. Structure and 532 Infrastructure Engineering, 7(6), 389-413.

533 Gaspar, P., de Brito, J. (2008) Service life estimation of cement-rendered façades.

534 Building Research and Information, 36(1), 44-55.

535 Gaspar, P. (2009) Service life of constructions: development of a method to estimate the 536 durability of construction elements, Application to renderings of current buildings (in 537 Portuguese), Instituto Superior Técnico, Technical University of Lisbon, Portugal. 538 (Doctor Thesis in Sciences of Engineering).

539 Gaspar, P. \& Brito, J. de (2011) Limit states and service life of cement renders on 540 façades. Journal of Materials in Civil Engineering, 23(10), 1396-1404.

541 Genrich, H.J. \& Lautenbach, K. (1981) System modelling with high-level Petri nets.

542 Theoretical Computer Science, 13, 109-136.

543 Girault, C. \& Valk, R. (2002) Petri nets for systems engineering: a guide to modeling, 544 verification, and applications. Springer: Verlag New York.

545 Hawk, H. \& Small, E.P. (1998) The BRIDGIT bridge management system. Structural 546 Engineering International, IABSE, 8(4), 309-314.

547 Hertlein, B. (1999) Predictive maintenance - what should be in a condition database. $8^{\text {th }}$ 
548 International Conference on Durability of Buildings and Components, Vancouver,

549 Canada, pp. 1203-12.

550 Kalbfleish, J. D. \& Lawless, J. F. (1985) The analysis of the panel data under a Markov 551 assumption. Journal of the American Statistical Association, 80(392), 863-871.

552 Kallen, M. J. \& van Noortwijk, J.M. (2006) Statistical inference for Markov 553 deterioration models of bridge conditions in the Netherlands. Third International 554 Conference on Bridge Maintenance, Safety and Management (IABMAS).

555 Korjenic, A., Zach, J. \& Hroudová, J. (2016) The use of insulating materials based on 556 natural fibers in combination with plant façades in building constructions. Energy and 557 Buildings, 116, 45-58.

558 Le, B., \& Andrews, J. (2016). Petri net modelling of bridge asset management using 559 maintenance-related state conditions. Structure and Infrastructure Engineering, 12(6), $560 \quad 730-751$.

561 Le, B., \& Andrews, J. (2015). Modelling wind turbine degradation and maintenance. 562 Wind Energy.

563 Leigh, J. M. \& Dunnett, S. J. (2016). Use of Petri nets to model the maintenance of 564 wind turbines. Quality and Reliability Engineering International, 32(1), 167-180.

$565 \mathrm{Li}, \mathrm{H}$. (1998) Petri net as a formalism to assist process improvement in the construction 566 industry. Automation in Construction, 7(4), 349-356

567 Man, K. F., Tang, K. S. \& Kwong S. (1999). Genetic algorithms: concepts and designs. 568 Springer-Verlag London, Great Britain.

569 Marsan, M. A., Conte, G. and Balbo, G. (1984) A class of generalized stochastic Petri 
570 nets for the performance evaluation of multiprocessor systems. ACM Transactions on

571 Computer Systems, 2(2), 93-122.

572 Marsan, M. A. \& Chiola, G. (1987) On Petri nets with deterministic and exponentially

573 distributed firing time. In Rozenberg, G., editor, Advances in Petri Nets 1987, volume

574266 of Lecture Notes in Computer Science, pages 132-145. Springer Berlin Heidelberg.

575 Marsan, M.A., Balbo, G., Conte, G., Donatelli, S. \& Franceschinis, G. (1994)

576 Modelling with generalized stochastic petri nets. Wiley Series in Parallel Computing,

577 John Wiley and Sons, West Sussex, UK, 1994.

578 MatLab (2016, $\quad$ February $\quad 5) . \quad$ Retrieved from

579 http://www.mathworks.com/help/gads/how-the-genetic-algorithm-works.html.

580 Mayer, P. \& Bourke, K. (2005) Durability rankings for building component service life

581 prediction, $10^{\text {th }}$ international Conference on Durability of Building Materials and

582 Components, Lyon, France, 546-553.

583 McDuling, J.J. (2006). Towards the development of transition probability matrices in 584 the Markovian model for predicted service life of buildings. $\mathrm{PhD}$ Thesis in Civil 585 Engineering, Faculty of Engineering, Built Environment and Information Technology, 586 University of Pretoria, Pretoria.

587 Molinero, C., \& Núñez, M. (2011). Planning of work schedules through the use of a 588 hierarchical multi-agent system. Automation in Construction, 20(8), 1227-1241.

589 Molly, M.K. (1982) Performance analysis using stochastic Petri nets. IEEE 590 Transactions on Computers, C-31(9): 913-917.

591 Morcous, G. (2006). Performance prediction of bridge deck systems using Markov 
592 chains. Journal of Performance of Constructed Facilities, 20(2), 146-155.

593 Morcous, G. \& Lounis, Z. (2005) Maintenance optimization of infrastructure networks

594 using genetic algorithms. Automation in construction, 14(1), 129-142.

595 Murata, T. (1989) Petri nets: Properties, analysis and applications. Proceedings of the 596 IEEE, 77(4), 541-580.

597 National Statistics Institute (INE), National Statistics - Census, 598 http://www.ine.pt/prodserv/quadro/mostraquadro 2001 (in Portuguese).

599 Ortiz-García, J.J., Seósamh, B.C. \& Martin, S.S. (2006) Derivation of transition 600 probability matrices for pavement deterioration modelling. Journal of Transportation 601 Engineering, 132(2), 141-161.

602 Paulo, P. V., Branco, F., \& de Brito, J. (2014). BuildingsLife: a building management 603 system. Structure and Infrastructure Engineering, 10(3), 388-397.

604 Paulo, P., Branco, F., de Brito, J., \& Silva, A. (2016). BuildingsLife - the use of genetic 605 algorithms for maintenance plan optimization. Journal of Cleaner Production, 121, 8460698.

607 Peterson, J.L. (1977) Petri nets. Computing Surveys, 9(3), 223-252.

608 Petri, C.A. (1962). Kommunikation mit Automaten. Bonn: Institut für Instrumentelle

609 Mathematik, Schriften des IIM Nr. 3. Also, English translation, Communication with

610 Automata. New York: Griffiss Air Force Base. Technical report RADC-TR-65-377,

611 volume 1, supplement 1, 1966.

612 Rama, D., \& Andrews, J. (2013). A system-wide modelling approach to railway 613 infrastructure asset management. In Proceedings of the $20^{\text {th }}$ Advances in Risk and 
614 Reliability Technology Symposium (pp. 7-22).

615 Rama, D., \& Andrews, J. (2016). Railway infrastructure asset management: the whole616 system life cost analysis. IET Intelligent Transport Systems, 10(1), 167-180.

617 Ramamoorthy, C.V. \& Ho, G.S. (1980) Performance evaluation of asynchronous 618 concurrent systems using Petri nets. IEEE Transactions on Software Engineering, SE$6196(5), 440-449$.

620 Rinke, N., von Gösseln, I., Kochkine, V., Schweitzer, J., Berkhahn, V., Berner, F., 621 Kutterer, H., Neumann, I. \& Schwieger, V. (2017). Simulating quality assurance and 622 efficiency analysis between construction management and engineering geodesy. 623 Automation in Construction, 76, 24-35.

624 Shohet, I., Puterman, M. \& Gilboa, E. (2002) Deterioration patterns of building 625 cladding components for maintenance management. Construction and Economics, $62620(4), 305-314$.

627 Shohet, I. \& Paciuk, M. (2004) Service life prediction of exterior cladding components 628 under standard conditions. Journal of Construction Management and Economics, 629 22(10), 1081-1090.

630 Silva, A., Dias, J.L.R., Gaspar, P.L. \& de Brito, J. (2013) Statistical models applied to 631 service life prediction of rendered façades. Automation in Construction, 30, 151-160.

632 Silva, A., Gaspar, P.L. \& de Brito, J. (2014) Durability of current renderings: A 633 probabilistic analysis. Automation in Construction, 44, 92-102.

634 Silva, A., Neves, L.C., Gaspar, P.L. \& de Brito, J. (2015). Probabilistic transition of 635 condition: render façades. Building Research \& Information, 44(3), 301-318. 
636 Silva, A., Gaspar, P.L. \& de Brito, J. (2016) Comparative analysis of service life

637 prediction methods applied to rendered façades. Materials and Structures, DOI $638 \quad 10.1617 / \mathrm{s} 11527-016-0832-6$.

639 Schneeweiss, W.G. (2004) Petri net picture book (an elementary introduction to the 640 best pictorial description of temporal changes). LiLoLe-Verlag GmbH, Germany.

641 Tang, F., Guo, M., Dong, M., Li, M. \& Guan, H. (2008) Towards context-aware 642 workflow management for ubiquitous computing. International conference on 643 embedded software and systems (ICESS'08), 221-228.

644 Thompson, P., Small, E., Johnson, M. \& Marshall, A. (1998) The Pontis bridge 645 management system. Structural Engineering International, IABSE, 8(4): 303-308.

646 Uzam, M., Gelen, G., Saleh, T.L. (2015) Think-globally-act-locally approach with 647 weighted arcs to the synthesis of a liveness-enforcing supervisor for generalized Petri 648 nets modeling FMSs. Information Sciences; In Press, 1-26.

649 Van der Aalst, W.M. (2002) Making work flow: On the application of Petri nets to 650 business process management. Application and theory of petri nets, Springer, 1-22.

651 Wang, J. (2012). Timed Petri nets: Theory and application (Vol. 9). Springer Science \& 652 Business Media.

653 Wang, S., \& Xie, J. (2002). Integrating Building Management System and facilities 654 management on the Internet. Automation in construction, 11(6), 707-715.

655 Wang, K. \& Zaniewski, J. (1996) 20/30 hindsight: The new pavement optimization in 656 the Arizona state highway network. Interfaces, 26(3), 77-89.

657 Wong, J. K., \& Li, H. (2009). Development of intelligence analytic models for 
658 integrated building management systems (IBMS) in intelligent buildings. Intelligent 659 Buildings International, 1(1), 5-22.

660 Yianni, P.; Rama, D.; Neves, L.; Andrews, J. \& Castlo, D. (2017), A Petri-Net-based 661 modelling approach to railway bridge asset management, Structure and Infrastructure 662 Engineering, 13(2), 287-297.

663 Yianni, P.; Neves, L.; Rama, D.; Andrews, J.; Dean, R. (2016) Incorporating local 664 environmental factors into railway bridge asset management, Engineering Structures, $665128,362-373$, ISSN 0141-0296, http://dx.doi.org/10.1016/j.engstruct.2016.09.038.

666 Zhang, D., Hu, H., \& Roberts, C. (2017). Rail maintenance analysis using Petri nets. 667 Structure and Infrastructure Engineering, 13(6), 783-793.

668 Zuberek, W.M. (1980) Timed Petri nets and preliminary performance evaluation. In 669 Proceedings of the $7^{\text {th }}$ annual symposium on Computer Architecture, New York, USA, $670 \quad 88-96$. 
673 Figure 1 - Illustrative example of the degradation conditions of rendered façades (photographs by Gaspar, 674 2009)

675 Figure 2 - Example of a Petri net including three places, and one transition

676 Figure 3 - An example of the Petri net scheme of the deterioration model

677 Figure 4 - Procedure for computing the probability of occurrence of the observed transition

678 Figure 5 - Procedure for optimization of the parameters of the probability distributions (adapted from 679 Morcous and Lounis, 2005)

680 Figure 6 - Comparison of the predicted future condition profile over time for both deterioration models:

681 (a) average condition; (b) standard deviation of condition

682 Figure 7 - Comparison of the predicted future condition profile over time for all probability distribution 683 analysed: (a) mean condition; (b) standard deviation of condition

684 Figure 8 - Probabilistic distribution of all degradation condition levels over time: (a) Level A (black) and

685 B (grey); (b) Level C (black) and D (grey); (c) Level E

686 


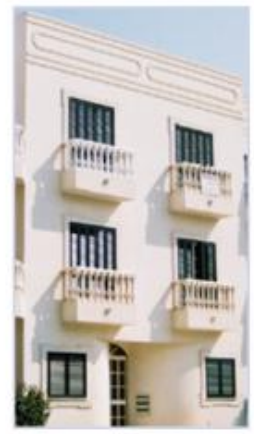

Level A

$\mathrm{Sw}<1 \%$

688

689

690

691

692

693

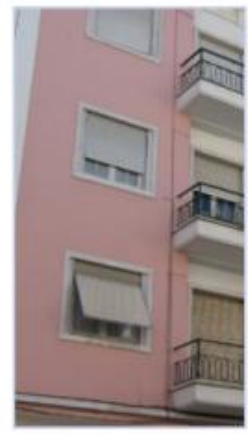

Level B

$1 \% \leq \mathrm{Sw}<5 \%$

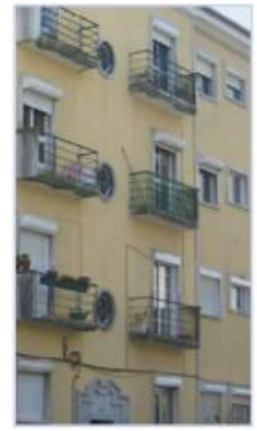

Level C

$5 \% \leq \mathrm{Sw}<15 \%$

Figure 1

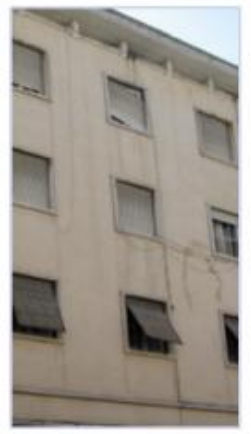

Level D

$15 \% \leq \mathrm{Sw}<30 \%$

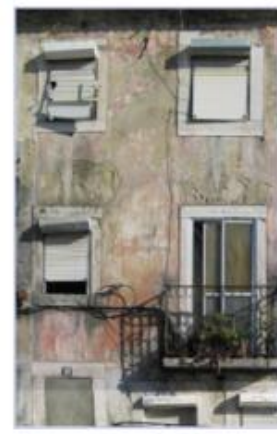

Level E $\mathrm{Sw} \geq 30 \%$

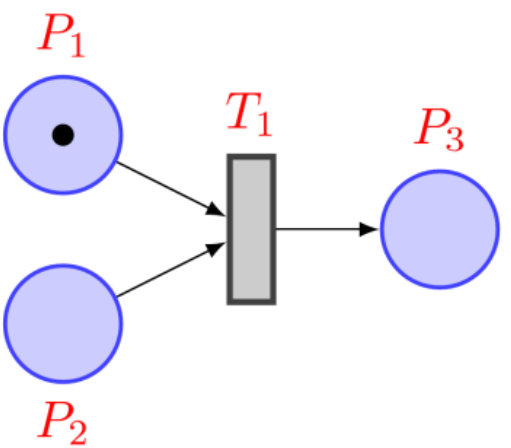

Figure 2

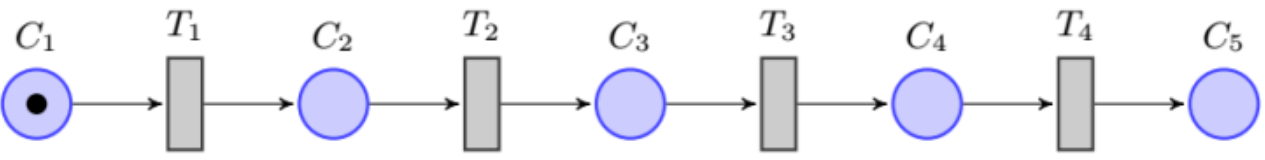

Figure 3 


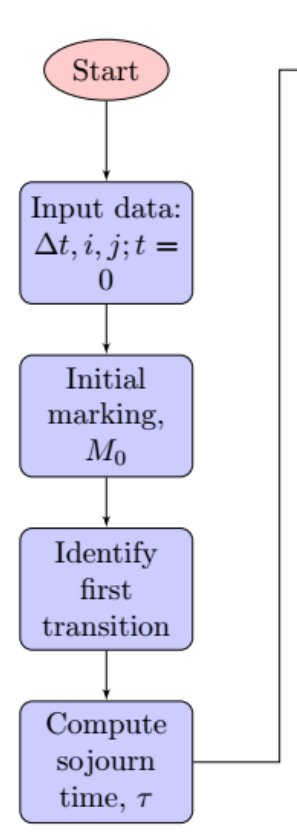

695

696

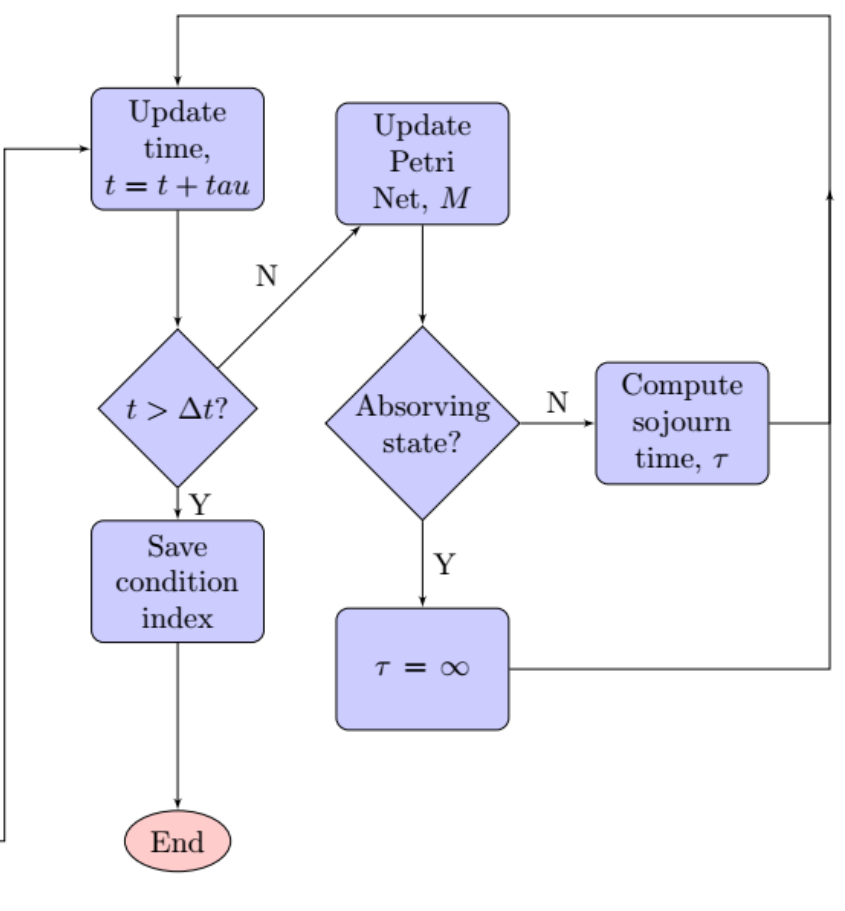

Figure 4

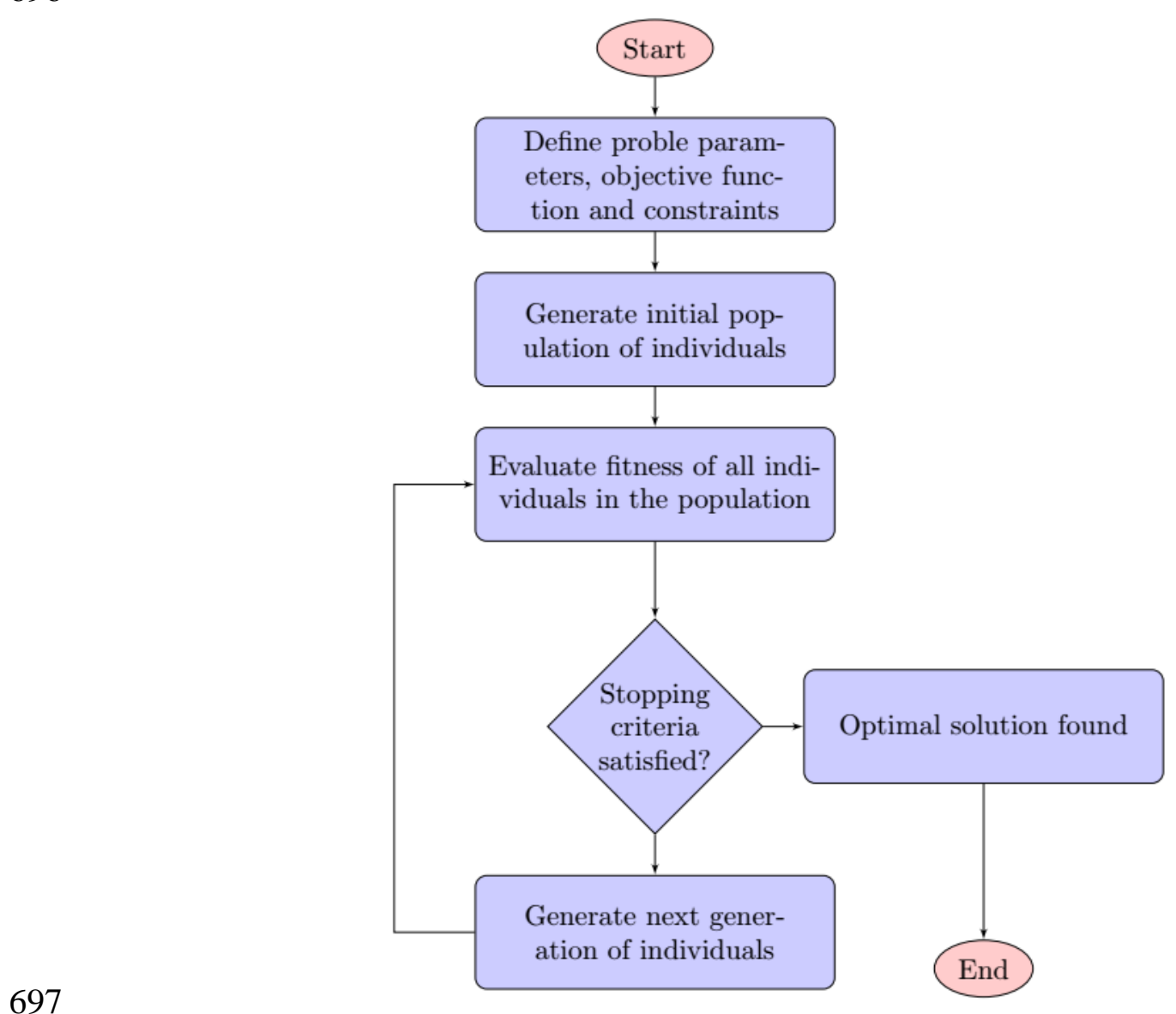


699

701

702

703

704

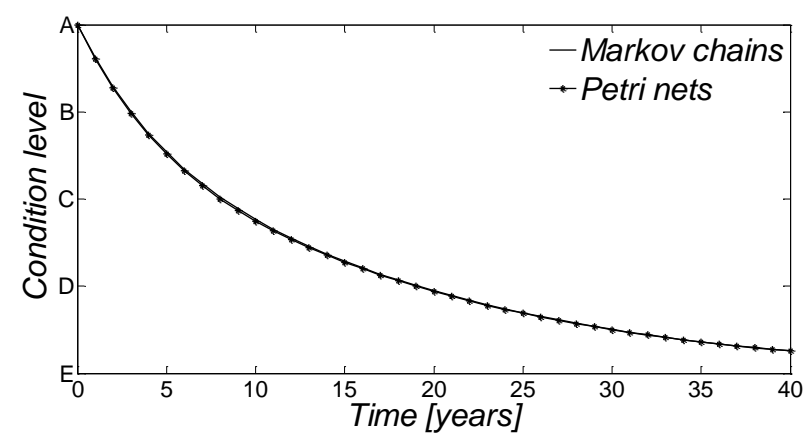

(a)

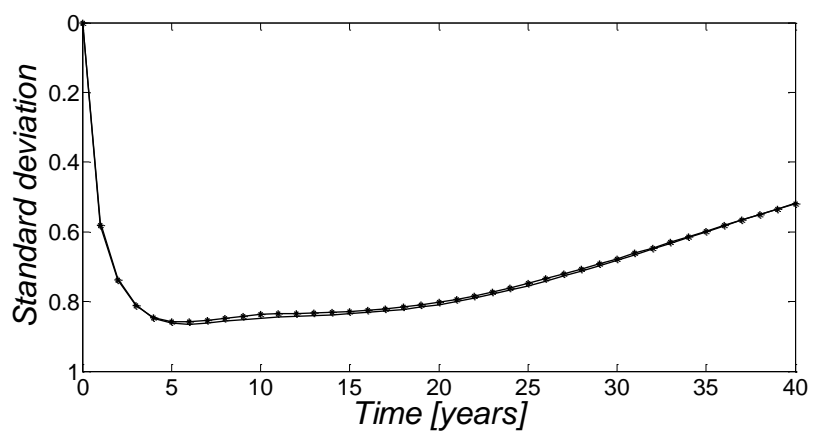

(b)

Figure 6

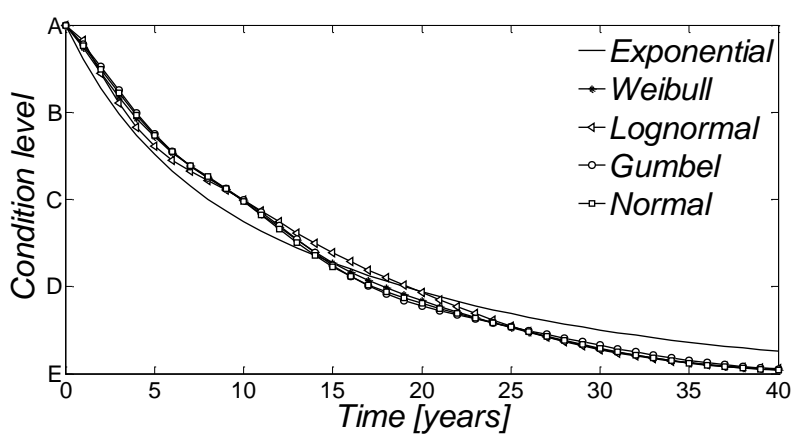

(a)

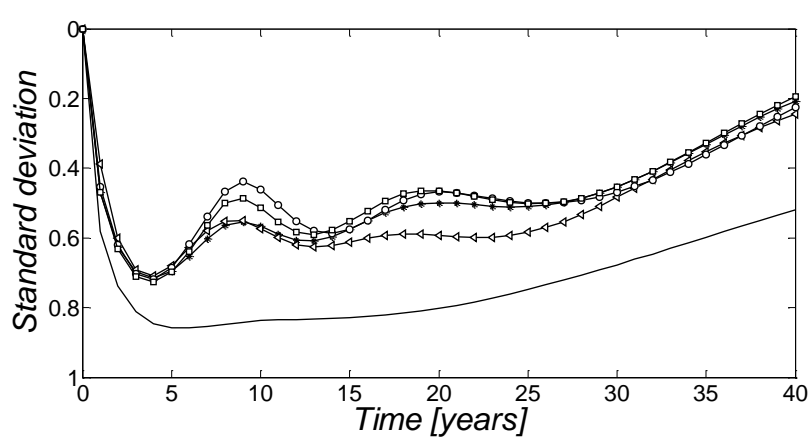

(b)

Figure 7 
710

711

712

713

714

715

716

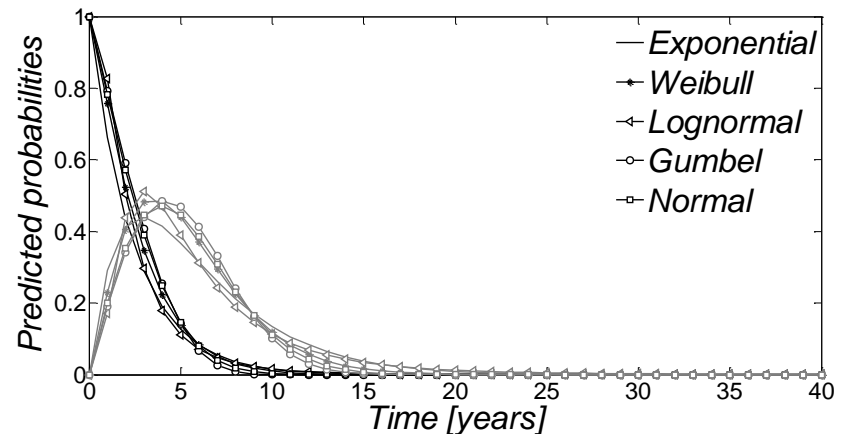

(a)

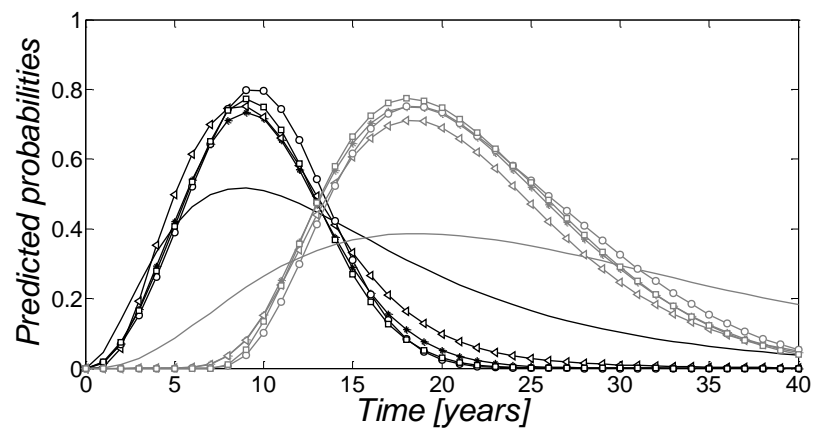

(b)

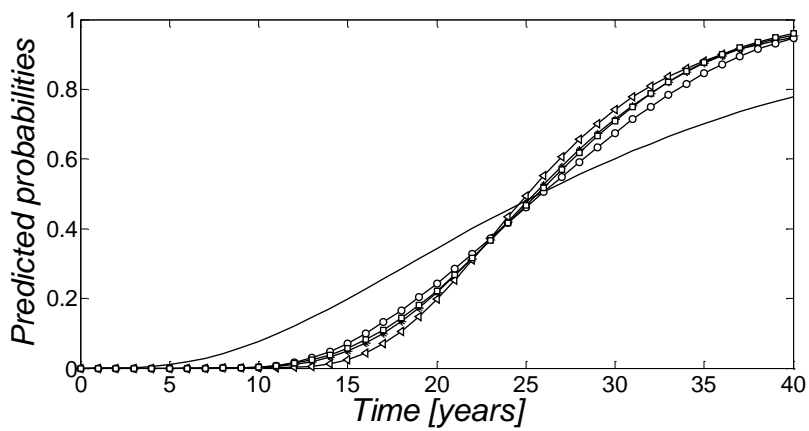

(c)

Figure 8 
719 Table 1 Description of the degradation conditions of rendered façades

720 Table 2 - Comparison of the optimal parameters of the Markov chains and Petri net models

721 Table 3 - Number of observed and predicted coating on each degradation condition for both models

Table 4 - Optimal parameters obtained in all probability distribution analysed

723 Table 5 - Number of observed and predicted coating in each condition level for each probability distribution

724 Table 6 - Mean error [\%] obtained for each probability distribution

725 Table 7 - Optimal parameters obtained for Weibull 3-parameters distribution

726 Table 8 - Number of observed and predicted coating for Weibull 3-parameters distribution

727 


\begin{tabular}{|c|c|}
\hline $\begin{array}{l}\text { Condition } \\
\text { level }\end{array}$ & Description \\
\hline $\begin{array}{l}\text { Condition } \\
\text { A }\end{array}$ & $\begin{array}{l}\text { Most favourable condition. Complete mortar surface with no visible } \\
\text { degradation, with uniform colour, showing no dirt or detachment }\end{array}$ \\
\hline $\begin{array}{l}\text { Condition } \\
\quad \mathrm{B}\end{array}$ & $\begin{array}{l}\text { Mortar with a non-uniform surface with likelihood of localized voids } \\
\text { determined by percussion, but no signs of detachment. Small cracking } \\
(0.25 \mathrm{~mm} \text { to } 1.0 \mathrm{~mm}) \text { in localized areas and changes in the general colour } \\
\text { of the surface might exist. Eventual presence of microorganisms. }\end{array}$ \\
\hline $\begin{array}{l}\text { Condition } \\
\quad \mathrm{C}\end{array}$ & $\begin{array}{l}\text { Mortar with localized detachments or perforations, revealing a hollow } \\
\text { sound when tapped and detachments only in the socle, with easily visible } \\
\text { cracking ( } 1.0 \mathrm{~mm} \text { to } 2.0 \mathrm{~mm} \text { ) and showing dark patches of damp and dirt, } \\
\text { often with microorganisms and algae. }\end{array}$ \\
\hline $\begin{array}{l}\text { Condition } \\
\quad \mathrm{D}\end{array}$ & $\begin{array}{l}\text { Mortar with an incomplete surface due to detachments and falling of } \\
\text { mortar patches, showing wide or extensive cracking }(\geq 2 \mathrm{~mm}) \text { and very } \\
\text { dark patches with probable presence of algae. }\end{array}$ \\
\hline $\begin{array}{l}\text { Condition } \\
\text { E }\end{array}$ & $\begin{array}{l}\text { Most serious condition, requiring an immediate corrective action, } \\
\text { associated with incomplete mortar surface due to detachments and falling } \\
\text { of mortar patches. Also revealing a wide or extensive cracking }(\geq 2 \mathrm{~mm}) \text {, } \\
\text { with very dark patches and probable presence of algae. }\end{array}$ \\
\hline
\end{tabular}

Table 2

\begin{tabular}{lccccc}
\hline \multirow{2}{*}{ Model } & \multicolumn{4}{c}{ Optimal parameters } & \multirow{2}{*}{ Likelihood } \\
\cline { 2 - 5 } & $\boldsymbol{\alpha}_{\mathbf{1}}$ & $\boldsymbol{\alpha}_{\mathbf{2}}$ & $\boldsymbol{\alpha}_{\mathbf{3}}$ & $\boldsymbol{\alpha}_{\mathbf{4}}$ & \\
\hline Markov chains & 0.4016 & 0.2819 & 0.0994 & 0.0761 & 82.4245 \\
\hline Petri net (Exponential) & 0.4201 & 0.2743 & 0.0966 & 0.0804 & 82.2582 \\
\hline${ }^{1}$ Data adapted from Silva et al. (2015) & & & &
\end{tabular}

731

Table3

\begin{tabular}{cccccc}
\hline \multirow{2}{*}{$\begin{array}{c}\text { Degradation } \\
\text { condition }\end{array}$} & \multirow{2}{*}{ Observed } & \multicolumn{2}{c}{ Predicted } & \multicolumn{2}{c}{ Error [\%] } \\
\cline { 2 - 6 } & & Markov chains $^{1}$ & Petri net & Markov chains & Petri net \\
\hline Level A & 13 & 12.57 & 12.17 & 3.3 & 6.4 \\
Level B & 18 & 17.77 & 17.63 & 1.3 & 2.1 \\
Level C & 31 & 28.64 & 28.96 & 7.6 & 6.6 \\
Level D & 15 & 17.29 & 17.47 & 15.3 & 16.5 \\
Level E & 22 & 22.74 & 22.77 & 3.3 & 3.5 \\
\hline
\end{tabular}

${ }^{1}$ Data sourced from Silva et al. (2015) 


\begin{tabular}{|c|c|c|c|c|c|c|}
\hline \multicolumn{2}{|c|}{ Parameters } & Exponential & Weibull & Lognormal & Gumbel & Normal \\
\hline \multirow{8}{*}{ 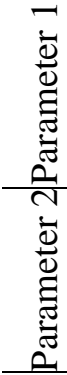 } & $\alpha_{1}$ & 0.4201 & 2.8616 & 0.7001 & 0.6112 & 0.4811 \\
\hline & $\alpha_{2}$ & 0.2743 & 3.8199 & 0.8702 & 1.5270 & 1.3919 \\
\hline & $\alpha_{3}$ & 0.0966 & 7.9483 & 2.0754 & 7.8258 & 7.2940 \\
\hline & $\alpha_{4}$ & 0.0804 & 14.1976 & 2.3615 & 11.4260 & 11.6725 \\
\hline & $\beta_{1}$ & - & 1.2149 & 0.7435 & 4.2326 & 3.2519 \\
\hline & $\beta_{2}$ & - & 1.4040 & 0.8572 & 4.4394 & 3.4303 \\
\hline & $\beta_{3}$ & - & 6.0816 & 0.3077 & 0.4219 & 0.1330 \\
\hline & $\beta_{4}$ & - & 2.0100 & 0.4612 & 11.7718 & 7.6393 \\
\hline $\mathrm{Li}$ & hood & 82.2582 & 70.4602 & 70.2610 & 70.4666 & 70.1237 \\
\hline
\end{tabular}

736

Table 5

\begin{tabular}{ccccccc}
\hline & & Level A & Level B & Level C & Level D & Level E \\
\hline Observed & 13 & 18 & 31 & 15 & 22 \\
& Exponential & 12.17 & 17.63 & 28.96 & 17.47 & 22.77 \\
$\vec{\Xi}$ & Weibull & 13.58 & 18.62 & 29.30 & 15.49 & 22.01 \\
$\vec{\Xi}$ & Lognormal & 13.09 & 17.58 & 32.07 & 14.67 & 21.60 \\
$\vec{\Xi}$ & Gumbel & 14.12 & 18.34 & 29.95 & 14.53 & 22.06 \\
$\vec{D}$ & Normal & 14.11 & 18.06 & 29.38 & 15.27 & 22.18 \\
\hline
\end{tabular}

738

Table 6

\begin{tabular}{lccccc}
\hline & Level A & Level B & Level C & Level D & Level E \\
\hline Exponential & 6.4 & 2.1 & 6.6 & 16.5 & 3.5 \\
Weibull & 4.4 & 3.5 & 5.5 & 3.3 & 0.0 \\
Lognormal & 0.7 & 2.3 & 3.4 & 2.2 & 1.8 \\
Gumbel & 8.6 & 1.9 & 3.4 & 3.2 & 0.3 \\
Normal & 8.5 & 0.4 & 5.2 & 1.8 & 0.8 \\
\hline
\end{tabular}

740

Table 7

\begin{tabular}{lcccc}
\hline Parameters & $i=1$ & $i=2$ & $i=3$ & $i=4$ \\
\hline$\alpha_{i}$ & 1.3998 & 2.5269 & 4.5874 & 1.4221 \\
$\beta_{i}$ & 0.7026 & 0.8977 & 1.8966 & 0.4718 \\
$\gamma_{i}$ & 0.8803 & 0.7551 & 4.1532 & 7.7902 \\
\hline Likelihood & 69.0345 & & & \\
\hline
\end{tabular}

742

Table 8

\begin{tabular}{lccccc}
\hline & Level A & Level B & Level C & Level D & Level E \\
\hline Observed & 13 & 18 & 31 & 15 & 22 \\
Weibull 3-parameters & 12.96 & 17.68 & 32.04 & 14.31 & 22.01 \\
Mean error [\%] & 0.3 & 1.8 & 3.3 & 4.6 & 0.0 \\
\hline
\end{tabular}

